\title{
Detectors for in vivo range and dose verification in proton therapy
}

\author{
R. Alarcon*, D. Blyth, E. Galyaev, J. Holmes, L. Ice and G. Randall \\ Department of Physics, Arizona State University \\ Tempe, Arizona 85287-1504, USA \\ "ralarcon@asu.edu \\ M. Bues ${ }^{\dagger}$ and M. Fatyga \\ Mayo Clinic Arizona \\ Phoenix, Arizona 85054, USA \\ †bues.martin@mayo.edu
}

Published 1 September 2016

\begin{abstract}
Particle detection instrumentation to address the in vivo verifications of proton dose and range is under development as part of a proton therapy research program focused on patient quality assurance. For in vivo proton range verification, a collimated gamma detector array is under construction to indirectly measure the position of the Bragg peak for each proton beam spot to within 1-2 mm precision. For dose flux verification, a proton fluence detector based on the technology of the Micromegas is under construction. This detector has an active area of about $100 \mathrm{~cm}^{2}$, coordinate resolution of better than $1 \mathrm{~mm}$, and handling of incident proton beam fluxes of $10^{9}-10^{13}$ particles/s.
\end{abstract}

Keywords: Proton therapy; proton range; particle detectors.

\section{Introduction}

Proton radiation therapy is a powerful technique for the treatment of various types of cancer where localized tumors have been properly diagnosed. Using the fact that protons deposit most of their energy at the end of their range, the radiation delivered can be mostly limited to the tumor region with minimum possible damage to the surrounding healthy tissue. Dose distributions in proton radiation therapy are computed within software applications called Treatment Planning Systems that use a Computed Tomography scan of the patient as an input for a radiation transport algorithm. Accurate prediction of dose distributions requires precise knowledge of the range of a pencil beam in tissue. Uncertainty in the actual range of a beam is one of the most significant limitations on the accuracy of treatment planning and thus an important limitation on the accuracy of proton therapy. Experimental verifications of the actual dose and proton

This is an Open Access article published by World Scientific Publishing Company. It is distributed under the terms of the Creative Commons Attribution 3.0 (CC-BY) License. Further distribution of this work is permitted, provided the original work is properly cited. 
range during treatment are essential information for patient quality assurance (QA) programs and for the optimization of the powerful simulation techniques. ${ }^{1}$

Mayo Clinic is building state of the art proton beam therapy facilities in Rochester, Minnesota and in Phoenix, Arizona, employing pencil beam scanning systems that are more accurate than other forms of radiotherapy (including all first generation proton therapy systems based on passive scattering technology). As part of a research program focused on patient QA, we are developing new particle detection instrumentation to address the in vivo verifications of proton dose and range. For proton range verification, we are developing a collimated gamma detector array to indirectly measure the position of the Bragg peak for each spot (as in active scanning) to within 1-2 mm precision. For dose flux verification, we are developing a proton fluence detector based on the technology of the "Micromegas" (Micro-MEsh Gaseous Structure) ${ }^{2}$ with active area of no less than $100 \mathrm{~cm}^{2}$, coordinate resolution of better than $1 \mathrm{~mm}$, and handling of incident proton beam fluxes of $10^{9}-10^{13}$ particles/s. The status of the design and construction of these developments is presented below.

\section{Detector for Range Determination}

The main advantage of proton beam therapy for cancer is that protons deposit more of their energy at the end of their range than at the patient's surface. In order to exploit this advantage, it is necessary to precisely control the proton range. This allows radiation oncologists to increase the dose to the tumor while reducing the dose to healthy tissue. On the other hand, there are some practical limitations in minimizing normal tissue complications and improving tumor control due to range uncertainties and the proximity of critical organs close to the tumor.

One way to facilitate more precise treatment plans is to verify the proton range in vivo by detecting the gamma rays produced by the nuclear interactions of the proton beam within the patient. These gamma rays include prompt photons emitted during the nuclear reactions and delayed emission from the decay of unstable nuclear reaction products. The main objective of utilizing these nearly instantaneous prompt gamma rays will be to image the dose deposition, which demands significant research and development for bringing this technique to clinical practice.

The interpretation of prompt gamma ray spectra depends critically on the knowledge of the nuclear interactions between the protons and patient tissue. Decades of protonnucleus scattering studies have produced a vast amount of data and the development of robust phenomenological models to interpret the many reaction channels. These models may be limited in the correct interpretation of interactions of protons with human tissue, but nonetheless represent the best analysis from nuclear physics and are now embedded in comprehensive computer simulation codes such as Monte Carlo N-Particle (MCNP) and Geant $4 .^{3}$ 


\subsection{Simulations}

Using the work of Ref. 4 as our baseline, we have simulated a collimated array of CsI detectors using both MCNP and Geant4. The results from both codes agree very well, and those derived from Geant 4 are presented here. In Fig. 1 (left), the geometry of the detector arrangement is shown with tracks of gamma rays being emitted by the impingement of $120 \mathrm{MeV}$ protons on a water target. Each CsI $(6 \mathrm{~cm}$ tall by $9 \mathrm{~cm}$ deep by $0.5 \mathrm{~cm}$ thick) crystal is positioned behind a lead collimator $(6 \mathrm{~cm}$ tall by $10 \mathrm{~cm}$ deep by $0.2 \mathrm{~cm}$ thick) with a water phantom along the beam direction (z-axis). Figure 1 (right) shows as a function of $\mathrm{z}$ the number of detected gamma rays above $2 \mathrm{MeV}$ when $5 \times 10^{7}$ protons of $120 \mathrm{MeV}$ stop on the water target. Also shown is the Bragg curve for 120 $\mathrm{MeV}$ on the same water target.
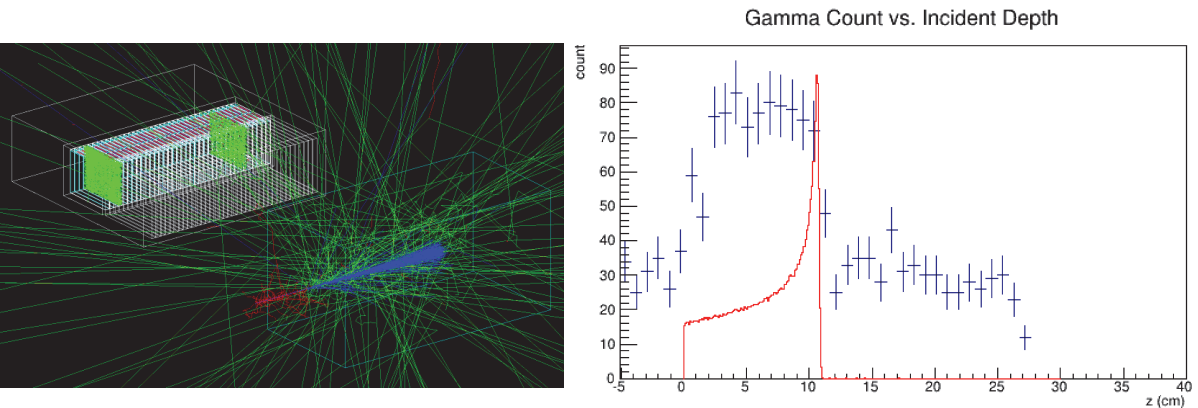

Fig. 1. Left: Array of collimated CsI in Geant4. Right: number of detected gammas above $2 \mathrm{MeV}$ as a function of the incident depth for $120 \mathrm{MeV}$ protons with the Bragg curve.

The above simulations were carried out with the Geant4 Physics List QGSP_BIC_HP with similar results obtained for protons ranging from 80 to $200 \mathrm{MeV}$. Neutrons were not included in the results presented in Fig. 1, but they tend to make less clear the distal edge observed at the position of the Bragg peak. These results were corroborated with the MCNP code, and as such, they are being used as a benchmark for the design and building of the actual detector.

\subsection{Detector prototype}

In order to rely on the Geant 4 simulation as a benchmark for the full array design, the simulation must be calibrated. A CsI(Tl) single-crystal prototype was constructed and tested as a mean of calibrating the simulation and also to study the feasibility of pulse shape discrimination in $\mathrm{CsI}(\mathrm{Tl})$ between gammas and neutrons. The scintillation crystal has dimensions $6 \mathrm{~mm}$ x $90 \mathrm{~mm}$ x $70 \mathrm{~mm}$. The crystal is coupled via Nyogel OC-440 optical gel to two Hamamatsu S12572-025 multi-pixel photon counters (MPPC), each having an active area of $9 \mathrm{~mm}^{2}$ for detecting the scintillation photons. Each MPPC signal is amplified and sent to separate digitizer channels. The crystals are wrapped with diffusively reflective Teflon (PTFE), and the crystal/diode assembly sits in an aluminum casing. An identical setup was modeled in Geant4. 


\subsubsection{Simulation calibration}

To calibrate the simulation, a ${ }^{60} \mathrm{Co}$ source was placed at various positions about the detector as shown in Fig. 2. Coincident signals between both MPPC channels above 1 photoelectron equivalent (PE) were digitized. The analysis of these data was to integrate both waveforms for one event and histogram the percentage of the total signal that each channel contributed into separate histograms. Figure 3 (left) shows that the most probable percentage of the total scintillation signal for the MPPC nearest the source was about $60 \%$. For comparison an equivalent histogram (Fig. 3 (right)) is then generated using Geant 4 results, where the ${ }^{60} \mathrm{Co}$ is modeled as an isotropic mono-energetic gamma source $(1.25 \mathrm{MeV})$. The amount of statistics for the simulated data is adequate for extracting a peak value of the fraction of total scintillation light.

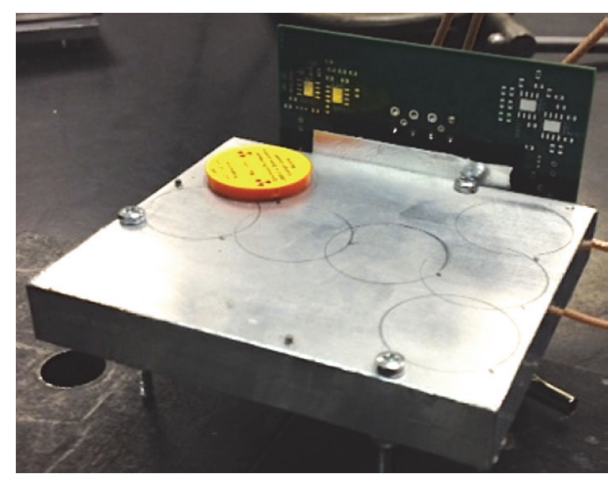

Fig. 2. Positions of the ${ }^{60} \mathrm{Co}$ source used for simulation calibration.
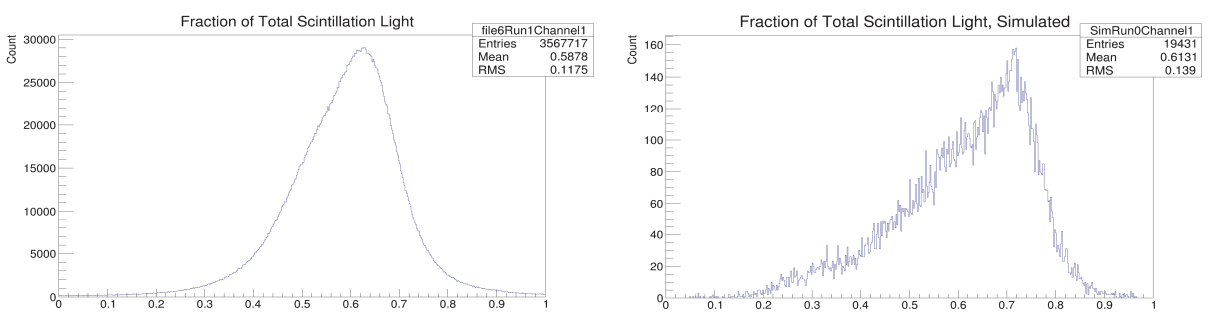

Fig 3. Left: A histogram of the percentage of the event signal for the setup shown in Fig. 2. Right: An equivalent plot generated from Geant4 data.

The calibration procedure is to then vary the optical parameters in the simulation until a good match is found between the peaks of simulation results and prototype detector data. The simulation has shown the most probable percentage of the total signal is highly sensitive to the Teflon reflectivity and to a lesser extent, the CsI (Tl) transmission length. Ongoing work includes modifying the simulation until the peak positions between the prototype data and the simulated data are in agreement for each source position. 


\subsubsection{Pulse shape discrimination in $\mathrm{CsI}(\mathrm{Tl})$}

One important aspect of measuring the Bragg Peak location lies in being able to discriminate prompt gammas from the background neutrons. One method for discriminating is to analyze the pulse shapes. Scintillation pulse shapes are often accurately modeled as a combination of fast and slow decaying exponentials. To this end the setup shown in Fig. 2 has been tested using a ${ }^{241} \mathrm{AmBe}$ source, which produces neutrons and gammas at similar intensities.

Figure 4 shows the average scintillation pulse shape (properly de-convolved from the preamp response) of the prototype detector response to ${ }^{241} \mathrm{AmBe}$. The shape of Fig. 4 fits extremely well to two decaying exponentials as evidence of the fast and slow decay modes known to be present in $\mathrm{CsI}(\mathrm{Tl})$ scintillation crystals. In general, different particles will activate the scintillating channels in differing amounts. A common method for discriminating is to integrate the fast and slow parts of the scintillation waveform and then compare.

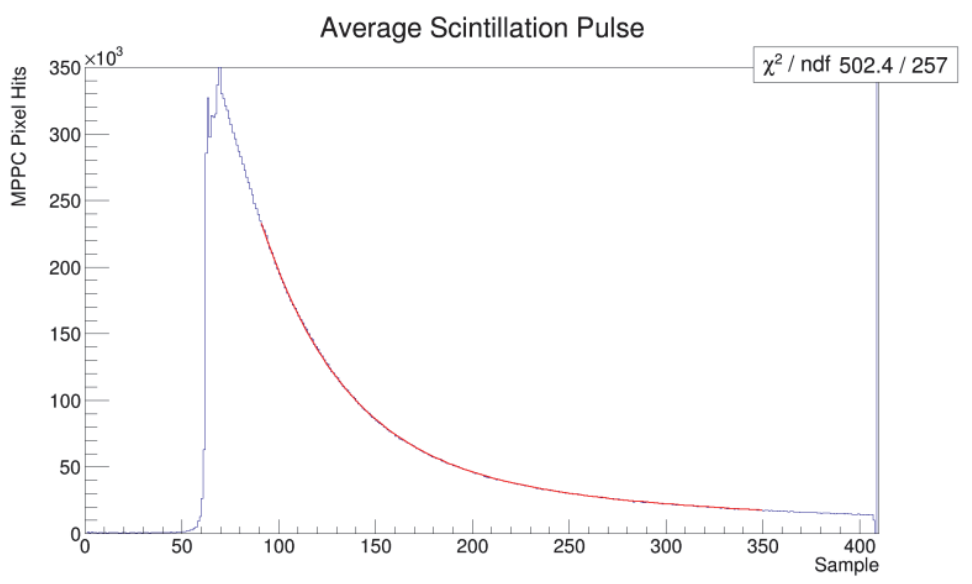

Fig. 4. After de-convoluting the preamp response from the scintillation pulse shape, 10,000 CsI (Tl) scintillation responses to a ${ }^{241} \mathrm{AmBe}$ source were averaged. The results show a good fit to two decaying exponentials.

Due to only having two small MPPCs on the crystal, a low number of PE are produced per energy deposited giving a large statistical variance in the number of fast $\mathrm{PE}$ and slow PE and the inability to discriminate the neutrons from the gamma rays. For this reason, another prototype detector utilizing 32 MPPCs per crystal and two crystals (each crystal same as previously described) is currently being produced to improve the PE statistics to better study the feasibility of pulse shape discrimination in CsI(Tl). This should increase the signal to noise ratio by a factor of four per crystal over previous results while also increasing the total neutron cross section by adding more scintillation material. 


\section{Dose Verification Detector}

An important component of the clinical use of a proton beam therapy facility is patient specific quality assurance (QA). QA is carried out for every patient and is an integral part of ensuring safety and efficacy of treatment. In a quality assurance procedure, every clinical proton beam is applied to a detector before being applied to the patient. To this end, we are embarking on the design and construction of such a detector based on a new radiation detector technology, the Micromegas. Since about 1992, at the CEA Saclay (France) and at CERN laboratories, the Micromegas technology ${ }^{2}$ has been developed in order to obtain more stable, reliable, precise and fast detectors. These detectors are now proven to be very robust and can be produced via a cost-efficient industrial process.

The Micromegas working principle is shown in Fig. 5 where the incident particle (in this case a proton) will ionize the gas atoms creating electron/ion pairs in low electric field region. Electrons drift towards a micromesh where they encounter a high electric field amplification gap and the avalanche effect is generated. The electronic signal is collected by charge amplifiers connected to the strips of the electrode read out.

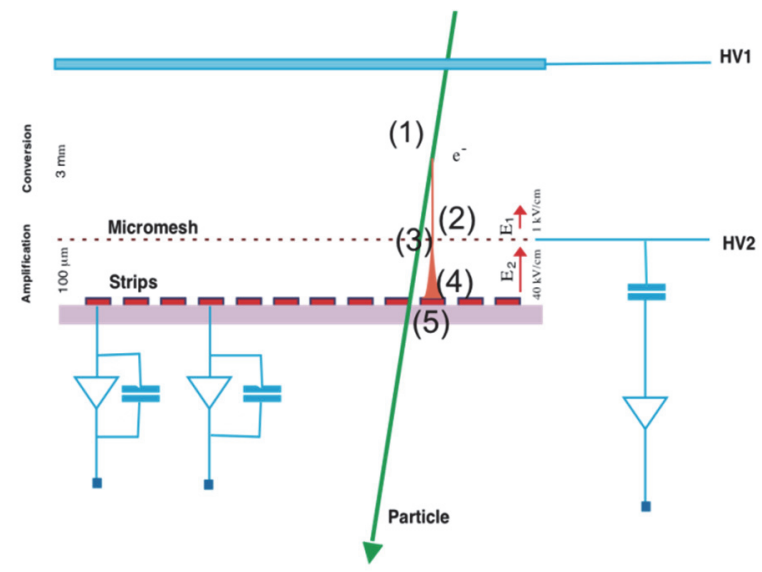

Fig. 5. A scheme of how the Micromegas detector works showing the incident particle (1), the initial electronion pairs (2), the micromesh (3), the avalanche region (4), and the electrode readout (5). Source: https://en.wikipedia.org/wiki/MicroMegas_detector\#/media/File:MMPrincipe.png (http://creativecommons.org/licenses/by-sa/3.0/).

\subsection{Design considerations}

The following are the main design considerations that were taken into account for choosing a proton fluence detector based on the technology of the Micromegas:

- A coordinate $(\mathrm{X}-\mathrm{Y})$ resolution of at least $1 \mathrm{~mm}$ for an array that covers the entire practically useful treatment aperture (up to $30 \times 30 \mathrm{~cm}^{2}$ ). 
- Capability for accurate fluence mapping measurements by capturing the absolute dose equivalent readings for proton beams.

- Radiation-hard technology that ensures prolonged lifetime over silicon arrays, diodes, and other competing solutions (estimated total exposures are to exceed 500 KGy).

- Fast, accurate, and absolute dose equivalency measurements with response linearity similar to conventional ionization chambers.

- Cost efficiency through considerable reduction of active electronic readout channels as compared to granulated arrays (diode, silicon) while maintaining high coordinate accuracy through transverse planar readout in $\mathrm{X}$ and $\mathrm{Y}$ coordinates, and resolving the ambiguities via the diagonal stereo readout layers.

- Structurally, the detector must be durable and mechanically stable for everyday use and handling.

- Production cost in labor and materials is a fraction of most competitive solutions, such as amorphous silicon arrays and diodes.

\subsection{Prototype construction}

The starting point to satisfy the most of the above considerations is to first build a prototype detector with an active area of about $10 \mathrm{~cm}$ by $10 \mathrm{~cm}$. We are in the process of implementing this first step.

\subsubsection{Pattern readout design}

We have chosen a 3-D readout system with hexagonal pixels arranged over an area of about $100 \mathrm{~cm}^{2}$. Figure 6 shows the schematic arrangement of the pixels. Three coordinates per pixel are available for readout. In this manner, about 240 electronic channels are used to read out about 7000 pixels covering the area of interest. The printed circuit board (PCB) design of the readout electrode is presented in Fig. 7. This is an ASIC/FPGA (Xilinx) board whose purpose is to integrate the signals, digitize them, and do a basic data reduction. The sampling rate can be adjusted to anything between 1 and $100 \mathrm{MHz}$. The detector will be readout at a rate of around $1 \mathrm{kHz}$.

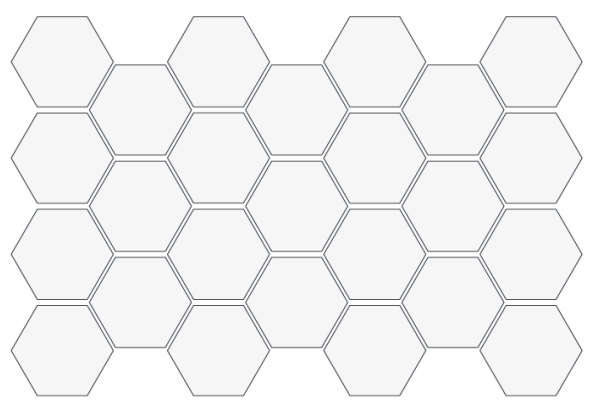

Fig. 6: Hexagonal pixel arrangement for the proton fluence Micromegas detector. 


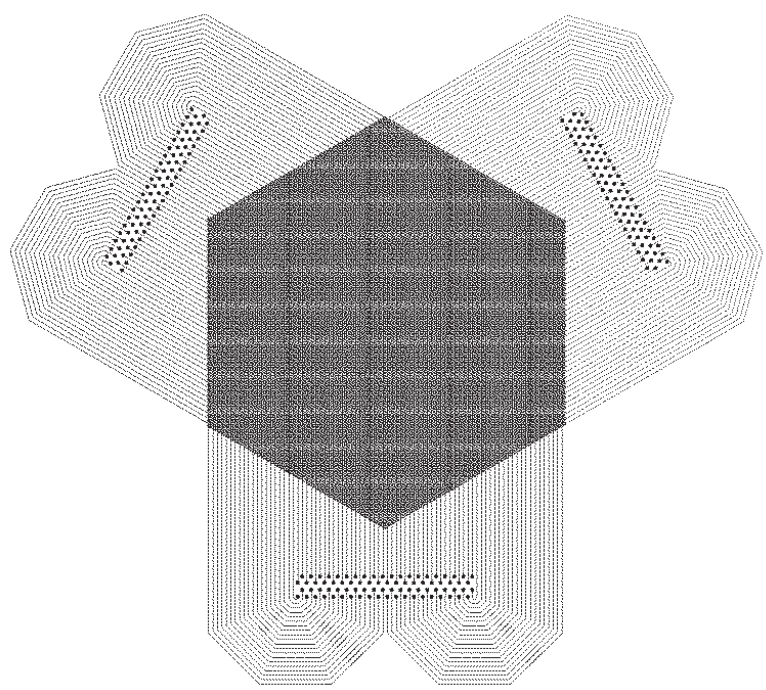

Fig. 7. Printed circuit board to be used as the electrode readout in the proton fluence detector.

\subsubsection{Mechanical design}

The construction proceeds by first building the PCB shown in the previous section. Attaching the micromesh to the PCB follows this. The detector is then enclosed in a gastight aluminum housing (see Fig. 8). The structure provides external connections for the 3-D readout of the pixels as well as connections for gas handling and HV connectors. The gas allows for a controlled ionization region. The incident particles will ionize gas molecules. Once the ionization electrons reach the amplification region, the ionization shower will occur. Without the gas, no natural amplification could occur. The gas will possibly be P10 (Argon with 10\% Methane), but this has not been confirmed. The gas would need to be chemically inert.

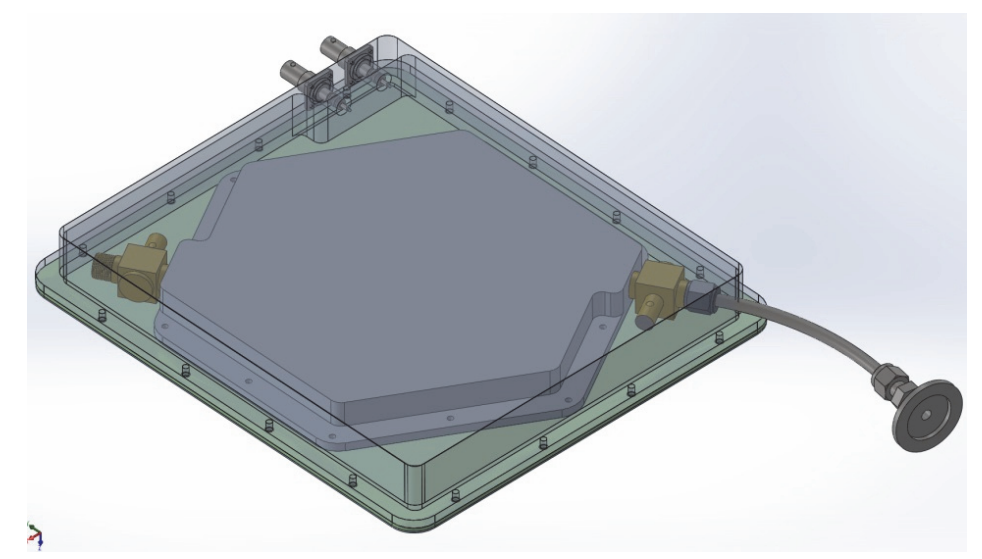

Fig. 8. Mechanical housing for the proton fluence detector showing power and gas connections. 


\section{Summary}

Detector designs were presented with the intended capability of verifying the proton range and the dose delivered during treatment. The motivation is due to the construction by Mayo Clinic of a new proton beam facility in Phoenix, Arizona. The emphasis is on patient QA.

For the proton range verification during treatment, the proposed detector is an array of $\mathrm{CsI}(\mathrm{Tl})$ scintillation crystals with a set of lead collimators in front of the crystals. Simulations for this array indicate that the range could be determined with a precision of about $2 \mathrm{~mm}$. In the actual detector, the CsI crystals are coupled with MPPCs for collecting the scintillation light. We have developed and tested an electronic readout system for the MPPCs, and optimization work is underway before full implementation into the final detector.

The proton fluence detector is based on the technology of the Micromegas. A prototype with an active area of about $100 \mathrm{~cm}^{2}$ is under construction. This device is implemented with a 3-D readout system of approximately 240 electronic channels for about 7000 pixels.

\section{References}

1. H. Paganetti (ed.), Proton Therapy Physics, Series in Medical Physics and Biomedical Engineering (CRC Press, 2012).

2. Y. Giomataris, Ph. Rebourgeard, J.P. Robert, and G. Charpak, Nucl. Instr. Meth. Phys. Res. A 376, 29 (1996).

3. MCNP, see https://monp.lanl.gov; Geant4 see http://geant4.cern.ch

4. C. H. Min et al., Med. Phys. 39, 4 (2012). 